\section{$\underset{\substack{\text { hommes } \\ \text { \& migrations }}}{ }$}

\section{Hommes \& migrations}

Revue française de référence sur les dynamiques

migratoires

$1313 \mid 2016$

1983, le tournant médiatique

\title{
La percée du Front National
}

\section{Alec G. Hargreaves}

\section{CpenEdition \\ Journals}

\section{Édition électronique}

URL : http://journals.openedition.org/hommesmigrations/3555

DOI : 10.4000/hommesmigrations.3555

ISSN : 2262-3353

\section{Éditeur}

Musée national de l'histoire de l'immigration

\section{Édition imprimée}

Date de publication : 1 janvier 2016

Pagination : 29-35

ISBN : 978-2-919040-34-6

ISSN : $1142-852 X$

\section{Référence électronique}

Alec G. Hargreaves, «La percée du Front National », Hommes \& migrations [En ligne], 1313 | 2016, mis en ligne le 01 janvier 2019, consulté le 16 mars 2020. URL : http://journals.openedition.org/ hommesmigrations/3555; DOI : https://doi.org/10.4000/hommesmigrations.3555 


\title{
LA PERCÉE \\ DU FRONT NATIONAL
}

par ALEC G. HARGREAVES, professeur émérite à l'université d'État de Floride.

\author{
En 1981, le FN n'avait même pas recueilli les 500 signatures \\ nécessaires pour présenter un candidat à l'élection \\ présidentielle. Deux ans plus tard, il fait une percée sans \\ précédent aux élections municipales. Face à l'échec de \\ la gauche devant la montée du chômage, Jean-Marie Le Pen \\ s'est employé à polariser le débat public sur son thème \\ de prédilection : l'immigration, présentée comme la cause \\ de tous les maux qui touchent le pays. Ses amalgames ont peu \\ à peu fait mouche dans des médias friands de polémiques. \\ Voici comment ses idées sont devenues audibles.
}

La percée électorale du Front national lors des élections municipales en 1983 marque incontestablement un tournant majeur dans la vie politique de la France d'après-guerre. Jusque-là l'extrême droite, délégitimée par la collaboration sous le régime de Vichy, avait peiné à retrouver une place dans le jeu électoral. Mais, pendant la campagne pour les élections municipales de mars 1983, le parti commence à faire parler de lui et cette tendance s'accentuera au cours de l'été. L'attention se focalise surtout sur Dreux, où le parti lepéniste gagnera $17 \%$ du vote lors du premier tour d'une élection partielle qui se tiendra au mois de septembre. ${ }^{1}$ Suite à cette percée au niveau local, le FN gagnera entre 10 et $18 \%$ du vote dans pratiquement toutes les élections nationales et européennes tenues jusqu'en 2012 et il deviendra le premier parti de France avec $28 \%$ du vote lors des régionales de
2015². Pourquoi ce basculement intervient-il en 1983 ? Quel a été le rôle des médias dans la montée de FN ? Quelles en ont été les conséquences à long terme?

\section{Un terrain préparé par d'autres partis}

Si le facteur le plus évident dans l'essor électoral du FN réside dans ses dénonciations des fléaux attribués à l'immigration, celles-ci ne suffisent pas pour expliquer la percée de 1983. Car si la stigmatisation des immigrés est fondamentale au discours du parti lepéniste depuis sa fondation en 1972, elle ne lui accorde pas le moindre profit électoral pendant les dix premières années de son existence. Pendant cette période, sans anticiper l'essor de l'extrême 
droite, les partis classiques lui préparent graduellement le terrain en jetant des suspicions sur les immigrés sans pour autant apporter des solutions aux problèmes qui leur sont associés et, en particulier, à celui qui, plus que tout autre, préoccupe lélectorat : la montée du chômage déclenchée par les chocs pétroliers des années 1970.

Quasi absente du débat politique pendant les Trente Glorieuses, l'immigration est parmi les premiers dossiers à retenir l'attention du président Giscard d'Estaing qui, dès son élection en 1974, interrompt les flux migra-

À quelques mois toires non européens dans de la présidentielle de 1981, l'espoir d'améliorer le mardes calculs électoraux dont les immigrés font les frais sont clairement présents dans ché de l'emploi. Sur l'avis du Conseil d'État, les regroupements familiaux reprendront, conformément aux obligations de la France en tant que signataire de la Convention européenne des droits de l'homme. Cependant, à partir de 1977, le gouvernement multiplie les efforts non seulement pour bloquer toute nouvelle immigration de main-d'œuvre non européenne mais aussi pour inciter ou forcer le rapatriement d'immigrés installés déjà en France, parmi lesquels les Algériens sont les premiers ciblés ${ }^{3}$. C'est l'époque de l'aide au retour, conçue pour encourager les immigrés à repartir dans leur pays, des projets de loi Bonnet-Stoléru visant à faciliter le rapatriement forcé de travailleurs immigrés sans emploi, et des expulsions administratives de jeunes délinquants étrangers, surtout algériens. À gauche, pour démontrer sa solidarité avec les ouvriers français qui constituent le noyau de sa base électorale, le Parti communiste tente de freiner l'accès des immigrés aux cités de HLM des municipalités de banlieue où le Parti a ses fiefs. À la veille de Noël 1980, le maire communiste de Vitry, Paul Mercieca, avec le soutien de Georges Marchais et du Comité central du PCF, fait bloquer à l'aide d'un bulldozer l'installation à Vitry de trois cents travailleurs maliens transférés de la commune voisine de Saint-Maur. Taxé de racisme, Paul Mercieca renvoie l'accusation contre le maire giscardiste de Saint-Maur, qu'il juge coupable d'un « coup de force raciste » en raison de son rôle dans le transfert des Maliens de Saint-Maur à Vitry ${ }^{4}$. Deux mois plus tard, le maire communiste de Montigny-lèsCormeilles, Robert Hue, sera à son tour accusé de racisme à la suite de sa dénonciation d'un Marocain soupçonné de trafic de drogue ${ }^{5}$. À quelques mois de la présidentielle de 1981, des calculs électoraux dont les immigrés font les frais sont clairement présents dans des escarmouches locales.

Ce thème reste secondaire au niveau national, où les élections de 1981 seront remportées par la gauche, grâce surtout à sa promesse de restaurer l'emploi en rompant avec le capitalisme et en initiant une importante politique de relance économique. Pendant la campagne électorale, les socialistes, qui tiendront l'Élysée et Matignon à partir de 1981, ont pris d'importants engagements destinés à favoriser l'insertion des populations d'origine immigrée, dont beaucoup seront mis rapidement en œuvre : droit d'association accordé aux étrangers, régularisation de 132000 sans-papiers, abrogation de l'aide au retour, interruption des expulsions administratives, facilitation des regroupements familiaux, initiatives en faveur des quartiers défavorisés, création des zones d'éducation prioritaire (ZEPs), etc. Mais, dans le domaine qui préoccupe plus que tout autre la masse des Français, loin de baisser, le chômage ne cessera de monter.

Au cours de l'hiver 1982-1983, l'industrie de l'automobile est touchée par une vague de grèves où les travailleurs immigrés sont particulièrement en vue. Exaspérés par ces troubles dans un secteur phare de l'économie nationale, des ministres socialistes affirment, sans avancer la moindre preuve, que les grévistes agissent sur la base de 
(C) AGENCE IM'MÉDIA.

motivations islamistes qui sont fondamentalement étrangères à la France. Selon le Premier ministre, Pierre Mauroy, les travailleurs immigrés chez Renault « sont agités par des groupes religieux et politiques qui se déterminent en fonction de critères ayant peu à voir avec la réalité sociale française $^{6} »$. Le ministre de l'Intérieur, Gaston Defferre, affirme plus spécifiquement qu' «il s'agit d'intégristes, de chi'ites », des termes qui viennent d'émerger dans le vocabulaire politique en France suite à la révolution islamiste menée par l'Ayatollah Khomeiny en Iran en $1979^{7}$. Les Iraniens
- chi'ites pour la plupart - sont en fait peu nombreux en France, où la majeure partie des musulmans (travailleurs immigrés maghrébins et africains assortis de leurs familles) sont des sunnites qui n'ont aucun lien avec les mouvements antioccidentaux évoqués par Gaston Defferre. Certes, les socialistes se montreront dans leur ensemble beaucoup mieux disposés que la droite envers les minorités immigrées. Il n'en reste pas moins que, par le biais de ces déclarations faites en janvier 1983, des ministres socialistes se distinguent comme les premiers politiciens de marque à stig- 
matiser les appartenances islamiques, réelles ou présumées, d'une importante part des populations immigrées ${ }^{8}$, un thème qui sera amplement repris dans les années à venir par d'autres partis, notamment le FN, dans d'interminables débats sur l'identité nationale.

\section{3, année charnière}

La médiatisation des mouvements sociaux dans l'industrie de l'automobile se mêle à un débat croissant sur le thème de l'immigration qui, pendant la campagne pour les élections municipales de mars 1983, deviendra un enjeu clé dans la lutte entre Gaston Deferre et Jean-Claude Gaudin pour la mairie de Marseille, ainsi que dans bien d'autres villes, telles que Dreux. Entre les deux tours du scrutin qui se soldera par une lourde défaite pour la gauche, un journaliste de La Semaine de l'émigration, organe de l'Amicale des Algériens en Europe, écrit : «Au fur et à mesure que la campagne électorale sétend et que la mobilisation des élec-

Le Pen martèle l'amalgame :

«immigration-insécuritéchômage ", qui est au cœur de sa campagne électorale,

à l’appui de laquelle il insère non pas un vrai-faux reportage mais un extrait d'un vrai reportage télévisuel réalisé par Rachid Arhab (qui à l'époque était un des rares journalistes d'origine maghrébine à la télévision française)

les chaines de montage, ni les meetings, ni les banderoles, ni les débrayages ne sont négligés. C'est que l'on veut à tout prix faire accréditer la thèse selon laquelle la crise de l'automobile est la faute des immigrés. De mémoire de téléspectateur, je n'ai pas vu, en dix ans, autant d'images d'immigrés à la télévision que depuis un mois $^{9}$ ! " La crise de l'automobile évoquée ici est double. D'une part, les suppressions de postes dans de nombreuses usines sont symptomatiques d'un très large affaiblissement de l'économie française depuis les chocs pétroliers des années 1970, qui ont été suivis par une forte montée du chômage. D'autre part, les lois Auroux, votées par la gauche en 1982 en vue de rééquilibrer les relations sociales au sein des entreprises, provoquent des conflits dans certains secteurs, notamment celui de l'automobile, où les travailleurs (parmi lesquels de nombreux immigrés) cherchent à faire respecter par les patrons les nouveaux droits que le gouvernement vient de leur accorder.

Les relations entre ces deux crises sont assez paradoxales. Alors que bien des politiciens affirment que les Français seraient moins touchés par le chômage si les travailleurs immigrés étaient moins nombreux, ces derniers sont en fait les plus durement touchés par les suppressions de postes dans des secteurs tels que l'automobile, où ils avaient été massivement recrutés pendant les Trente Glorieuses. Mais, là où ils sont en fait les premières victimes du chômage, ils sont souvent perçus comme les causes de celui-ci, et cela d'autant plus durant les grèves de 1982-1983 qui ont tendance à apparaître comme autant de coups portés à un secteur phare de l'économie française.

C'est sur ce genre d'amalgame que joue Jean-Marie Le Pen lors de l'une de ses premières interventions télévisées, une émission de campagne électorale réalisée par le FN dans le cadre de l'accès aux médias audiovisuels prévu par le code électoral pour les formations politiques participant aux municipales de 1983. Le format adopté au début de l'émission, diffusée le 2 février $1983^{10}$, est celui d'un simulacre d'interview dans laquelle JeanMarie Le Pen répond à des questions posées par Roland Gaucher, présenté comme " journaliste », qui est en fait un des cofondateurs du Front national, et qui en 1984 deviendra rédacteur en chef de Minute, très proche du FN. Tout au long de cette 
émission de propagande, Le Pen martèle l'amalgame : "immigration-insécurité-chômage ", qui est au cœur de sa campagne électorale, à l'appui de laquelle il insère non pas un vrai-faux reportage mais un extrait d'un vrai reportage télévisuel réalisé par Rachid Arhab (qui à l'époque était un des rares journalistes d'origine maghrébine à la télévision française). Rachid Arhab n'a bien entendu rien à voir avec les opinions politiques de JeanMarie Le Pen mais, à travers cette émission de propagande, on voit la facilité avec laquelle le leader du Front national exploite la couverture médiatique des grèves qui touchent le secteur automobile depuis l'automne de 1982, dans lequel les OS immigrés - surtout maghrébins et africains - sont particulièrement en vue. En sélectionnant des clips où ces derniers apparaissent dans des postures agressives, Le Pen dénonce «le tiers monde à l'assaut de la France et de l'Europe".

Dans l'immédiat, les bénéfices de cette campagne apparaissent modestes, avec l'élection de Le Pen au conseil du $20^{\mathrm{e}}$ arrondissement de Paris. Mais, six mois plus tard, le FN réalisera un score jusque-là sans précédent pour le parti en remportant $17 \%$ des suffrages lors du premier tour d'une élection partielle à Dreux, où la section locale du RPR, bastion de la droite «classique », fait alliance avec lui pour battre la gauche au deuxième tour du scrutin. Entre-temps, un changement majeur est intervenu dans la politique économique menée par la gauche, dont les pertes électorales en mars témoignent de l'évaporation de la confiance qui lui avait été accordée deux ans auparavant. Alors que le chômage augmente durant cette période, les finances publiques et la valeur du franc n'ont cessé de se dégrader. Quelques semaines après les municipales de mars 1983, le gouvernement se trouve contraint de prendre le tournant de la rigueur, scellant l'échec de ses promesses de relance économique et démontrant son impuissance, tout comme celle du gouvernement de centre-droit qui l'a précédé, à inverser la détérioration du marché de l'emploi.

\section{Les travailleurs immigrés au centre du débat politique}

Le tournant de la rigueur semble constituer à bien des égards le catalyseur qui permettra au FN de réaliser sa spectaculaire percée à Dreux à l'automne de 1983. Le parti lepéniste récolte, en effet, les fruits d'une conviction qui se propage à partir de 1983 au sein d'une partie de l'électorat, selon laquelle aucune des formations politiques classiques - de droite ou de gauche - ne semble capable d'apporter de solutions aux problèmes posés par la crise économique. Jusque-là, les luttes électorales avaient été dominées par une opposition entre la gauche et la droite basée sur des lignes de clivage pensées en termes de diffétraitent l'immigration comme un thème secondaire, le Front national est le seul à en faire le centre de son discours. En août 1983, parmi les Français interrogés par la Sofres, le renvoi des travailleurs immigrés chez eux est perçu solution proposée pour lutter contre le chômage.
Mais, là où les partis classiques comme la meilleure rences entre classes sociales tournant autour de luttes entre les forces du capital et du travail. Certes, en soulevant de différentes façons la question de l'immigration face à la crise qui sévit depuis les années 1970, des politiciens de pratiquement toutes les couleurs ont infiltré dans le discours politique une dimension ethnique. Mais, là où les partis classiques traitent l'immigration comme un thème secondaire, le Front national est le seul à en faire le centre de son discours. En août 1983, parmi les Français interrogés par la Sofres, le renvoi des travailleurs immigrés chez eux est perçu comme la meilleure solution proposée pour lutter contre le chômage ${ }^{11}$. Désormais, l’apparente impuissance de tous les partis classiques à restaurer l'emploi permet au parti lepéniste de faire valoir sa prétention à être le seul à proposer une solution valable, en recentrant l'ensemble du débat politique sur l'immigration présentée 
comme la cause principale de tous les fléaux affligeant le pays. En 1981, 43 \% des Français interrogés par la Sofres pensent que les notions de droite et de gauche sont pertinentes, contre $33 \%$ qui les trouvent dépassées. En 1984, les opinions se sont inversées : pour $49 \%$ des sondés, les notions de droite et de gauche sont dépassées, alors que seulement $37 \%$ les trouvent encore valables, et cet écart s'accentuera par la suite ${ }^{12}$. En 1991, sur quasiment toutes les questions, la majorité des sondés trouve qu'il y a peu de différences entre la gauche et la droite, la seule exception étant celle de l'immigration, sur laquelle $61 \%$ des interrogés affirment qu'il y a de grandes différences ${ }^{13}$. Au même moment, dans «l'idée que l'on se fait de la gauche ", la protection sociale arrive en tête mais, lorsqu'on passe à ce que les sondés perçoivent comme « la réalité de la gauche " au cours des dix dernières années, c'est-à-dire depuis la victoire de la gauche en 1981, c'est l'antiracisme qui prime sur toute autre notion ${ }^{14}$. La société française et les acteurs politiques qui cherchent à la gérer sont ainsi perçus à travers une grille de lecture où les différences ethniques prennent le pas sur les clivages socio-économiques, et tout laisse penser que 1983 a été l'année charnière dans ce basculement.

\section{Le rôle des médias}

Quel a été le rôle des médias dans la montée électorale du Front national ? Face aux succès remportés par le parti lepéniste au niveau national à partir de 1984, certains affirmeront qu'il faut lui barrer l'accès aux médias afin de réduire sa popularité électorale. Mais l'idée selon laquelle il y aurait une corrélation directe entre la présence médiatique du FN et la popularité du parti paraît peu convaincante. À plusieurs reprises, la très large médiatisation de déclarations antisémites faites par Le Pen est suivie par une baisse, provisoire au moins, de sa popularité $^{15}$. À d'autres moments, le FN est relativement peu présent dans les médias mais profite de la très forte couverture accordée à des controverses concernant les minorités arabes et musulmanes et d'autres thèmes qui sont étroitement associés à l'immigration dans le débat public. C'est le cas, par exemple, lors de l'affaire du foulard en 1989, dans la foulée de laquelle la candidate du FN remporte le deuxième tour d'une élection législative partielle à Dreux avec 61 \% du vote, ou encore lors de la campagne pour le premier tour de la présidentielle de 2002, quand le thème de l'« insécurité ", étroitement associé à la présence de populations d'origine immigrée, avait été massivement médiatisé ${ }^{16}$.

En octobre 1983, lorsque la Sofres demande pour la première fois si le FN représente un danger pour la démocratie, $43 \%$ des sondés répondent " non » tandis que $38 \%$ répondent « oui ». En mai 1984, les opinions sont partagées avec $44 \%$ de «non » contre $43 \%$ de « oui ». À partir d'octobre 1985, ceux qui voient le FN comme un danger pour la démocratie sont beaucoup plus nombreux que ceux qui sont de l'avis contraire (50\% contre $34 \%$ ) et cette tendance s'accentuera pendant les années suivantes pour atteindre $67 \%$ contre $25 \%$ en $1988^{17}$. Simultanément, les sans-réponses chutent de $19 \%$ en 1983 à $13 \%$ en 1984 et à $8 \%$ en 1988. En 1983, le parti lepéniste est relativement peu connu par le grand public, qui n'est donc guère en mesure de porter un jugement sur lui. À mesure que les Français deviennent mieux informés - grâce, essentiellement, aux médias -, une forte majorité d'entre eux affichent leur hostilité envers le FN, même si une importante minorité s'y rallie. L'adhérence de cette minorité aux idées de Le Pen semble être déterminée beaucoup moins par le niveau de la présence médiatique du FN que par sa focalisation sur les prétendus maux de l'immigration et sur la conviction qu'aucun autre parti n'est capable de les maîtriser.

Certes, le FN profite de profonds déséquilibres dans la représentation médiatique des populations d'origine immigrée qui sont déjà présents 
bien avant le tournant de 1983. Les immigrés sont depuis longue date cantonnés essentiellement dans des rubriques et des émissions d'informations et d'actualité, où ils tiennent notamment des rôles d'assistés, de délinquants et, depuis les chocs pétroliers des années 1970, de malvenus dans un marché de l'emploi en perte de vitesse ${ }^{18}$. Mais l'essor du FN à partir de 1983 ne serait probablement par survenu si des politiciens de pratiquement tous les bords - de gauche comme de droite - n'avait pas déjà jeté des suspicions sur les immigrés et si l'ensemble des formations politiques classiques n'avait pas fini par paraître aux yeux d'une importante partie de l'électorat toutes aussi impuissantes les unes que les autres face à la détérioration du marché de l'emploi.

\section{Conclusion}

Le tournant politique qui se manifeste dans le score réalisé par le Front national à Dreux au mois de septembre 1983 semble constituer à bien des égards le pendant d'un autre tournant - celui de la rigueur - que le gouvernement de la gauche a pris quelque mois plus tôt dans sa gestion des finances publiques, marquant l'échec des promesses de relance économique qui l'avaient porté au pouvoir en 1981. De façon moins abrupte, l'année 1983 constitue aussi le premier pas dans un troisième tournant politique dans la mesure où, par le biais de certains propos tenus par Pierre Mauroy et d'autres ministres socialistes, les travailleurs immigrés commencent à être repensés comme musulmans voire comme islamistes. L'absence de ce thème dans l'émission de campagne électorale diffusée de la part du Front national le 2 février, à peine quelques jours après les déclarations de Mauroy et de ses collègues, témoigne de la centralité de la question de l'emploi au moment où le parti lepéniste fait sa percée. Mais, au cours des années 1980, l'islam, perçu souvent à tort à travers le prisme de mouvements anti-occidentaux basés au Moyen-Orient, deviendra à son tour un enjeu central dans le débat entourant l'immigration, au point de faciliter le triomphe de la candidate frontiste à Dreux en décembre 1989 sur fond d'une massive médiatisation de la première affaire du foulard islamique.

Si les médias ont certainement une part de responsabilité dans la stigmatisation des minorités immigrées, ils n'ont tout de même pas créé les chocs pétroliers des années 1970, ni la montée du chômage et des sentiments d'insécurité provoqués par ceux-ci, ni les discours ni les actions de bien des politiciens de droite et de gauche qui, bien avant l'essor électoral du Front national, ont accusé les immigrés de causer ou d'aggraver ces problèmes. Relayés par les médias, ces discours stigmatisants sensibilisent le public au thème de l'immigration, dont le FN récoltera les fruits à partir 1983 chez une partie de l'électorat désormais convaincue qu'aucune des formations politiques classiques n'est capable de maîtriser la crise économique et le climat d'insécurité qu'elle engendre. 